\title{
RECONNECTIONLESS CME ERUPTION: PUTTING THE ALY-STURROCK CONJECTURE TO REST
}

\author{
L. A. Rachmeler ${ }^{1}$, C. E. DeForest ${ }^{2}$, And C. C. Kankelborg ${ }^{3}$ \\ ${ }^{1}$ University of Colorado at Boulder, Boulder, CO, 80304-0391, USA; laurel.rachmeler@colorado.edu \\ ${ }^{2}$ Southwest Research Institute, 1050 Walnut Street, Suite 300, Boulder, CO 80302, USA \\ ${ }^{3}$ Department of Physics, Montana State University, Bozeman, MT 59717, USA \\ Received 2008 August 11; accepted 2008 November 12; published 2009 March 9
}

\begin{abstract}
We demonstrate that magnetic reconnection is not necessary to initiate fast Coronal mass ejections (CMEs). The Aly-Sturrock conjecture states that the magnetic energy of a given force-free boundary field is maximized when the field is open. This is problematic for CME initiation because it leaves little or no magnetic energy to drive the eruption, unless reconnection is present to allow some of the flux to escape without opening. Thus, it has been thought that reconnection must be present to initiate CMEs. This theory has not been subject to rigorous numerical testing because conventional magnetohydrodynamics (MHD) numerical models contain numerical diffusion, which introduces uncontrolled numerical reconnection. We use a quasi-Lagrangian simulation technique to run the first controlled experiments of CME initiation in the complete lack of reconnection. We find that a flux rope confined by an arcade, when twisted beyond a critical amount, can escape to an open state, allowing some of the surrounding arcade to shrink, and releasing magnetic energy from the global field. This mechanism includes a true ideal MHD instability. We conclude that reconnection is not a necessary trigger for fast CME eruptions.
\end{abstract}

Key words: MHD - Sun: coronal mass ejections (CMEs) - Sun: magnetic fields

Online-only material: color figure

\section{INTRODUCTION}

Coronal mass ejections (CMEs), are large expulsions of magnetic field and plasma from the solar corona. The kinetic and gravitational potential energies contained in a $\mathrm{CME}$ are around $10^{31}-10^{32} \mathrm{erg}$, making these events some of the most energetic in our solar system (Forbes 2000; Hundhausen et al. 1994; Low 1990, 2001). It is thought that CMEs derive their energy from the magnetic field of the solar corona because this field is the only possible source for such a large reserve of energy (e.g., Forbes 2000; Klimchuk 2001; Low 1996).

The flux rope model is one possible pre-eruptive CME configuration. A flux rope is a length of magnetic field that has been twisted along its axis and is often held in place in the corona by an overlying arcade or ambient field. It is thought that cool photospheric plasma can become trapped in the center of a flux rope, creating a solar filament or prominence (van Ballegooijen \& Martens 1989; Ridgway et al. 1991; Priest et al. 1989). The flux rope configuration easily explains the clear threepart structure seen in many CMEs, specifically those associated with prominence eruptions (Low 1994; Gibson \& Low 1998). Because these structures are present in the low corona where magnetic field is strong and plasma density is low, they are magnetic-field-dominated. Recently, movies from Hinode have shown interesting dynamics that are not described by the flux rope model, which imply that at least some prominences are not low- $\beta$ (Berger et al. 2008), but the flux rope model remains useful. The lower coronal environment is frequently modeled as being force free because flow speeds are low, and $\mathbf{J} \times \mathbf{B}$ is the dominant force in the equation of motion. Gravity is also frequently ignored because it is a factor of $\sim 5$ weaker than the magnetic forces. Low- $\beta$ flux ropes are stable when the outward magnetic pressure force is balanced by an inwarddirected tension force. In models, an exterior arcade field is often added to increase the tension force and keep the flux rope from simply expanding in length and width as twist is added.
The approximate energy per unit length along the axis, $U$, stored in a Gold-Hoyle flux rope (Gold \& Hoyle 1960) that is finite in width is given by

$$
U=\frac{1}{8 \pi^{2}} \frac{\Phi^{2} b^{2}}{\ln \left(1+b^{2} R^{2}\right)},
$$

where $\Phi$ is the magnetic flux, $R$ is the radius of the tube, and $b$ is the twist parameter such that $\frac{B_{\phi}}{B_{z}}=b r$ (Sturrock et al. 2001). Although this equation is for a straight flux rope, we use it to approximate the energy per unit length of a curved flux rope whose ends are anchored on a flat photosphere. As twist accumulates, $b$ increases, $\Phi$ is constant and $R$ remains approximately constant (as long as the flux rope is confined by external field or pressure) so the total energy increases. When a flux rope that is not magnetically confined accumulates twist, its equilibrium state is expanded in length relative to the untwisted state so the twist per unit length does not necessarily increase with the total twist. Thus, flux ropes that are confined by an overlying arcade accumulate more energy because their length changes very little as $b$ increases.

The energy stored in magnetic fields is given by the volume integral of $B^{2} / 8 \pi$, up to conversion factors. The minimum energy of a magnetic system with a given photospheric boundary occurs in the potential, or vacuum field, configuration. As the field is stressed away from this configuration due to photospheric movements, the energy is increased above the evolving potential state by an amount commonly referred to as the magnetic "free energy." If the field reverts to the potential configuration, this free energy is released and in the case of solar active regions, is available to drive a CME. If the reconnection is localized and helicity is conserved, the lowest accessible energy state may not be potential, so the free energy is an upper boundary on the amount of energy that can be released. There is a global cap on the amount of energy that the magnetic field can provide. The magnetic virial theorem asserts that the total pressure force 


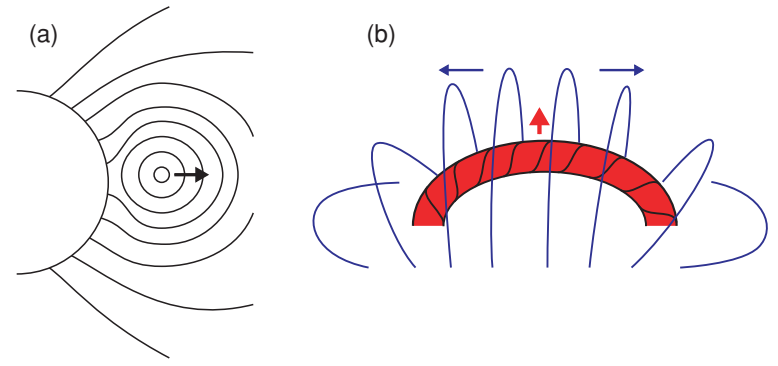

Figure 1. Behavior of flux rope expansion in 2.5D (a) and 3D (b). (A color version of this figure is available in the online journal.)

cannot exceed the tension force in a stable plasma environment (Priest 1982). The related Aly-Sturrock conjecture states that the global magnetic energy of a force-free field is at a maximum when the field is completely "open." This refers to magnetic flux that is anchored at the solar surface and extends radially outward a significant distance so that near the Sun, the field appears open (Aly 1984, 1991; Sturrock 1991). Many CME observations show prominences lifting off of the surface of the Sun, expanding to several solar radii, and leaving behind long radial field lines. If this conjecture is correct, then the implication is that CMEs which open large amounts of field must derive the bulk of their kinetic energy from sources other than the magnetic field because the field energy is actually greater in the post-CME configuration. Order of magnitude analysis has shown, however, that the magnetic field is the only source of energy that can potentially drive a $10^{32}$ erg CME (Forbes 2000). This poses a significant problem to ideal CME models. In an azimuthally symmetric $2.5 \mathrm{D}$ case, all of the field lines originally above a prominence-like feature would have to open to release the filament (Figure 1(a)). Thus, in the 2.5D case, to have an eruption that results in a net decrease of magnetic energy, reconnection must be present. There have been studies in which a closed 2.5D field is shown to have magnetic energy exceeding the open field energy when mass loading is present, but it has not been demonstrated that these fields can erupt without reconnection (Low 1996; Fong et al. 2002; Zhang \& Low 2004). In three dimensions, the flux rope is anchored in the photosphere, and the surrounding field can move away in the direction parallel to the flux rope axis (Figure 1(b)). Reconnection is not necessary in the fully three-dimensional (3D) case, as not all of the field must be open to have an eruption, only the flux rope opens, and thus the eruption is not necessarily relevant to the hypothesis of Aly and Sturrock because some of the field remains closed (Low 1986).

A related but more relevant question which has been asked is whether a configuration with some open force-free field can contain less energy than a configuration with the same boundary conditions that is fully closed (Low 1990). This question has been addressed semianalytically by Wolfson \& Low (1992) and Wolfson (1993), who showed that a fully closed field can contain magnetic free energy above the partially open field threshold, but they did not demonstrate a release mechanism for this energy. Likewise Choe \& Cheng (2002) found a fully closed 3D field that contains more energy than the open field limit, but that configuration is not accessible from an initial potential state with footpoint motions that conserve the boundary-normal field distribution. A demonstration of the ideal evolution of a field whereby free energy is first stored and then released, resulting in a partially open state, would once and for all eliminate the problem posed by the magnetic virial theorem and the AlySturrock conjecture in a fully $3 \mathrm{D}$ case.

Sturrock et al. (2001) described the possibility of driving CMEs with metastable magnetic fields. They analytically characterized one system in particular as a known metastable state: the previously mentioned twisted flux rope under an overlying arcade. This system is metastable because it is stable (due to the confining arcade) against small perturbations, but the energy of the erupted flux rope is lower than the contained flux rope. If the rope is tightly wound, it can open by pushing up locally on the arcade, decreasing the confining tension force, and escape by herniating through the weakened field, leaving the deflated arcade near the footpoints. The amount of twist needed is not unreasonable for the solar surface. Analytically, for a flux rope that is 10 times longer than its radius, the rope need only exceed 1.5 total turns about its axis to be in this metastable state (Sturrock 1991).

Numerous simulations exist which model flux rope CME initiation of the metastable configuration described above (e.g., Aulanier et al. 2005; Fan \& Gibson 2004; Titov \& Démoulin 1999; Török et al. 2004; Roussev et al. 2003). Most of these simulations have found that it is possible to herniate or reconnect to break through the arcade, but they do not exactly agree on how much twist is needed, or how unstable the resulting configuration is after the onset of writhe (helical geometry in the central field line) in the flux rope. Typically, these codes agree that the critical twist needed to erupt is around 1.5 turns, and that it is possible to get an eruptive event by twisting the footpoints of a flux rope.

CME initiation with these flux ropes has been modeled both with and without reconnection as the intended primary destabilizing factor. Theories that do not involve reconnection are referred to as ideal "loss of equilibrium" models (e.g., Roussev et al. 2003). The initial structure generally undergoes an ideal instability, such as the MHD kink instability, caused by a large amount of twist. Another possibility, if mass loading is critical in keeping the structure contained, is that mass displacement could upset the force balance and start the CME (Klimchuk 2001; Fong et al. 2002; Zhang \& Low 2004). Other theories, such as tether cutting (Moore \& Roumeliotis 1992) or "breakout" (Antiochos et al. 1999), explicitly include reconnection to decrease the strength of the overlying arcade. In models such as tether cutting and breakout, there are generally two stages of reconnection. Slow-Sweet-Parker style (Parker 1963)—reconnection occurs early in the evolution and destabilizes the system, allowing it to expand. This is often followed by fast-Petschek style (Petschek 1964)—reconnection that releases large amounts of energy in a short time and is believed to be the primary driver for fast, impulsive CMEs.

Essentially all existing numerical simulations of CME onset use Eulerian methods, in which a 3D grid of values is used. With magnetic fields (and indeed all vector fields and flows), sharp gradients are not conserved because derivatives are represented as finite differences. For magnetic fields, this means that the field will reconnect if gradients approach the size of the grid whether the modeler wishes it or not. Techniques such as adaptive mesh refinement can reduce the rate of numerical reconnection, but cannot remove it altogether. Hence it is not possible to separate the effects of ideal MHD evolution and magnetic reconnection with an Eulerian grid code. This can be problematic insofar as reconnection destabilizes a metastable system. By switching to a Lagrangian (field-aligned) formulation, we eliminate all reconnection, allowing study of ideal MHD instabilities (DeForest \& Kankelborg 2007). 


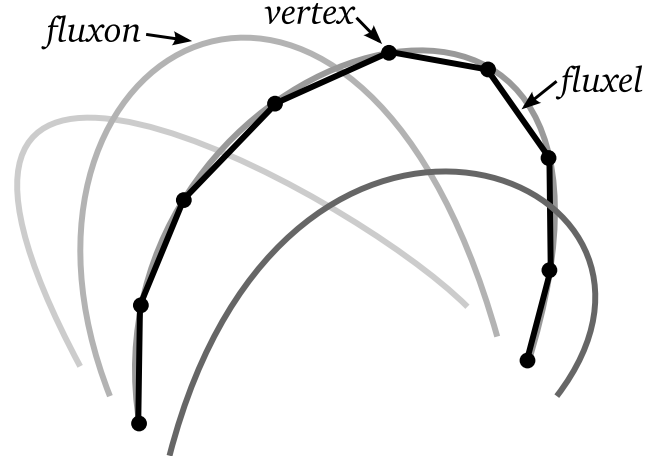

Figure 2. Geometry and nomenclature of the FLUX code. Finite-magnetic-flux field lines are called fluxons, which are broken into linear fluxel segments joined by vertices.

With our model, we are able to analyze simplified systems where topology is locked in and reconnection is not present. Note that we do not hypothesize that reconnection is not present in the Sun, only that to have a controlled numerical experiment, the effect of reconnection must be isolated, and we do this by eliminating it. Our method is unique in this way, and may offer insights that grid simulations cannot. In particular, we are able to demonstrate the existence of a true MHD instability that can release free energy into a $\mathrm{CME}$, even with no triggering reconnection.

\section{NUMERICAL MODEL}

\subsection{Computational Model}

The code used in this work is called FLUX (FieldLine Universal relaXer; DeForest \& Kankelborg 2007). This quasiLagrangian code represents a 3D field as a collection of fluxons, or field lines with finite magnetic flux. Each fluxon is broken into piecewise linear segments called fluxels, which are joined at vertex points (Figure 2). To reconnect, a fluxon must be explicitly broken and connected to another fluxon. With no reconnection, the code preserves magnetic topology; this is the case used in the current work. FLUX is coordinate free, so in order to compare the simulations with the Sun, we assume that our system is originally the size of an active region, which is a few tens of Mm across ( 1 spatial unit $=25 \mathrm{Mm}$ ). FLUX is under development and the version we used for this work is not a full MHD code. It does not include the effects of mass or plasma; thus it does not model dynamics. We are neglecting short-timescale changes of the system in favor of concentrating on the large-scale evolution.

FLUX computes nonlinear force-free equilibrium solutions from a prescribed initial topology and connectivity by balancing the components of the Lorentz force, which are resolved as a magnetic pressure and a magnetic tension force:

$$
0=-\nabla\left(\frac{B^{2}}{8 \pi}\right)+\frac{(\mathbf{B} \cdot \nabla) \mathbf{B}}{4 \pi}
$$

The tension force is computed from the geometry of the other fluxels on the same fluxon (e.g., the angle between successive fluxels), and the pressure is computed based on the geometry of the nearest surrounding neighboring fluxels. Each vertex is moved in the direction of net calculated force until the ratio of the net force to the sum of the magnitudes of the forces on each vertex is below a threshold level. Once all vertices are below this threshold, e.g., $0.1 \%$, the field is deemed to be in equilibrium.
(A more detailed mathematical description is available in DeForest \& Kankelborg (2007)).

Initial conditions in the code consist of a planar line-tied photosphere-like boundary with a set connectivity. The footpoint of each fluxon can be moved independently to simulate photospheric motions. After each footpoint movement, the field is allowed to relax to equilibrium before the next movement occurs. In this way, it is possible to create a quasi-static evolution of equilibrium states.

Current conservation is enforced by the geometry of the model; alpha (which varies between fluxons) is a quantity that can be computed from the model but is not explicitly tracked. The simulation does not consider the self-helicity of the flux tube represented by each fluxon and only mutual linking/helicity with adjacent fluxons is modeled; with sufficient fluxon density, self-helicity becomes negligible. Current is thus represented as geometrical twist between pairs of fluxons.

The simulation is bounded at the top by an open hemisphere. Fluxons that intersect this surface are free to move around on it. Closed loops that approach the surface are truncated and become two separate fluxons that then move independently. Open fluxons move to equalize magnetic pressure (there is no curvature on the final fluxel), which has the effect that the magnetic field at the upper boundary is normal to the surface.

\subsection{Simulation Set $U p$}

The simulated systems consist of a flux rope, an overlying arcade, and an outer ring of open field lines. Figure 3(a) shows this setup. The fluxons are tied to a planar lower boundary and evolve with a prescribed surface motion. The central flux rope is twisted incrementally in a solid-body-rotation pattern by four degrees each step and allowed to relax to equilibrium. The fluxrope footpoints are set at two spatial units apart, or $50 \mathrm{Mm}$.

One difficulty in examining these results has been with the energy calculation. Our code calculates the energy of every fluxel based on the cross-sectional area it occupies and its length. In regions where the fluxons are close to each other, this method works extremely well, but it has more trouble for the outermost fluxels in a system. We call this the "last-fluxon" problem and it is discussed by DeForest \& Kankelborg (2007). The volume that a last fluxel occupies is infinite, so it cannot be treated as small, violating the approximation used by the code. Once the system has herniated and expanded fully, the number of last fluxels is much greater, exaggerating the difficulty in determining the post-eruption energy. The open hemispherical surface at $35 R_{\odot}$ and an outer ring of fluxons were added to alleviate this problem. As a consequence of the surface, the field opens once it encounters the hemisphere and expands to fill the volume.

The footpoints of the outer ring remain stationary throughout the simulation. This outer ring is present to minimize the last fluxon problem because with the ring, none of the arcade or flux rope fluxels will be a last fluxel. The ring is positioned far from the rope and the arcade, about $2 R_{\odot}$ away, so it does not effect the evolution. The outer boundary at a radius of $35 R_{\odot}$ is much farther than the physical regime of applicability of FLUX. The transition to solar wind occurs at $\sim 4 R_{\odot}$ (Parker 1960; Kohl et al. 1997), so at most, our results have physical meaning up to this height.

We performed several simulations with varying numbers of fluxons in the flux rope and the arcade, each with the same basic setup. The flux rope consists of $9,16,25,36$, or 49 fluxons arranged in a square on the photosphere. The footprint of the 

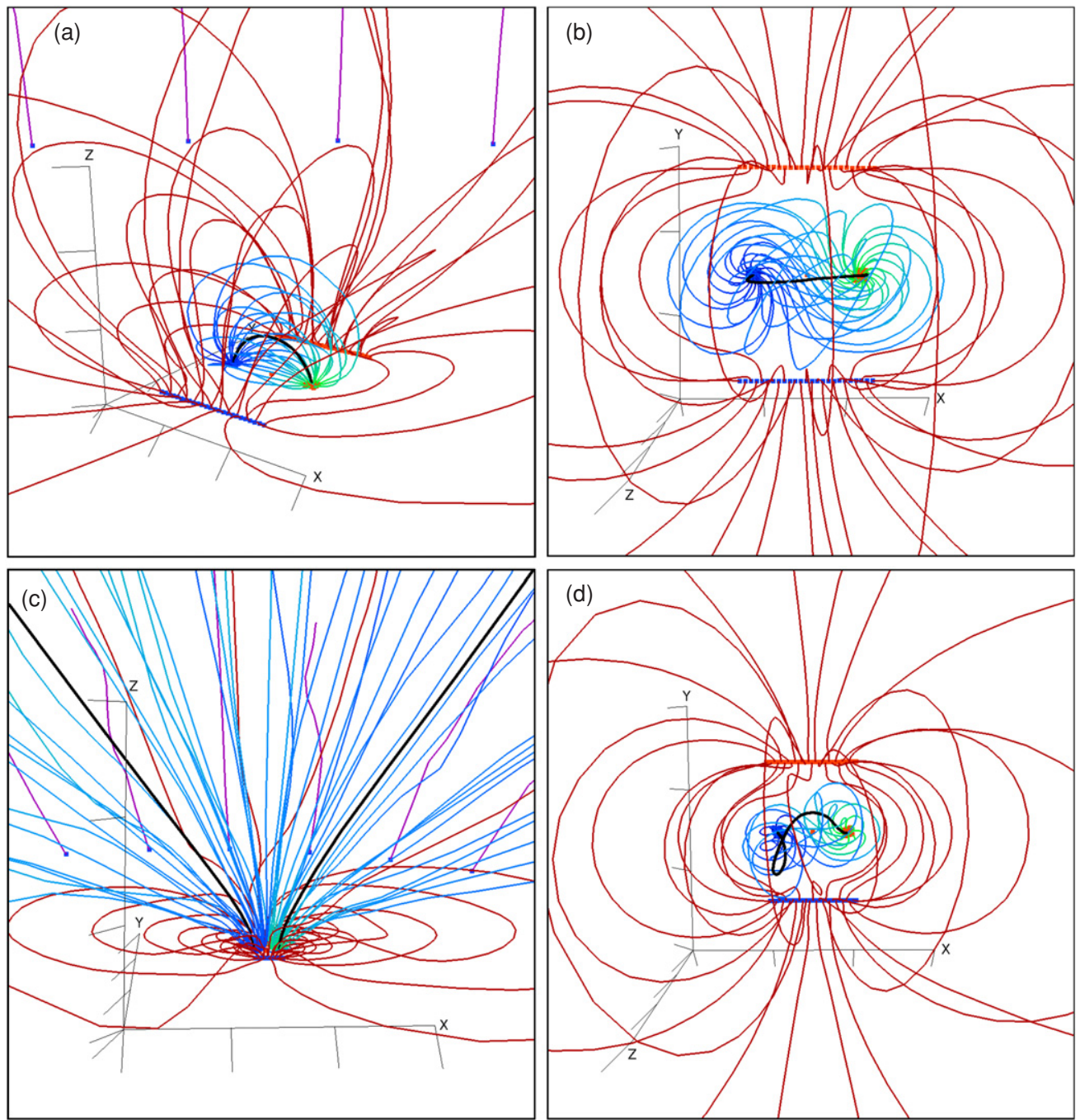

Figure 3. (a)-(c) Initial simulation setup and evolution of a system with 75 fluxons where the arcade is 25 fluxons shown in red and the flux rope is 25 fluxons in shown in a gradient from blue to green to show directionality. The central fluxon is in black to highlight the flux rope axis. The outer ring of 25 fluxons is shown in purple. (a) The equilibrium system with no twist, (b) with one turn, which has not kinked, (c) with 1.43 turns which has herniated and is open at the upper boundary. (d) Shows a different system with nine fluxons in the flux rope and three units of flux (27 fluxons) in the arcade at 2.1 turns, which demonstrates kinking without herniation. Note that the systems with one unit of arcade flux exhibit a slight kink while confined as evidenced by a small distortion in the central fluxon, but the systems with higher arcade flux exhibit much stronger kink before herniation.

flux rope is the same size in each case. For the rest of this paper, a unit of magnetic flux refers to the amount of magnetic flux associated with the flux rope, or the number of fluxons in the flux rope. In these simulations, the flux rope and the ring consist of one unit of flux, and the arcade contains one, two, or three units.

\section{SIMULATION RESULTS}

In all cases, we find that the flux rope herniates through the arcade after a certain amount of twist has been applied, entraining a few arcade fluxons with it as it goes. Figure 3(a)(c) demonstrates a typical sequence of events for the case of a 25 fluxon flux rope and a one-unit magnetic flux arcade. First, the flux rope twists about its central axis under the arcade. After about 1.4 turns have been applied to the flux rope (for one unit of arcade flux), the rope herniates. In this case, the flux rope does not significantly kink- the central axis remains mostly untwisted - but in the case of a stronger arcade, the flux rope does kink before it herniates. The stronger the arcade, the flatter the flux rope is, and the more twist is required to initiate herniation. Figure 3(d) shows a three-unit arcade system after it has undergone writhe; the black central fluxon is no longer straight. The onset of kink does not trigger herniation through the arcade. The flux rope continues to twist and writhe until it begins to herniate. In every case, after the onset of herniation, the flux rope expands rapidly while the arcade deflates. Figure 4 shows a plot of the height of the flux rope versus the twist imparted for various fluxon densities with one unit of arcade flux. The expansion occurs extremely rapidly, within one equilibrium step, once the flux rope breaks through the arcade.

We label this rapid expansion as an "eruption," because the size of the flux rope increases by a factor of 100 or more and breaks through the open surface in a single equilibrium time step, while releasing a nontrivial amount of energy. Thus, these large expansions are deemed eruptions and the flux rope fluxons are labeled as open.

The amount of twist needed to herniate through a given arcade strength varies with the number of fluxons used to represent the field (Figure 4); this may be indicative of grid effects that are setting an unstable twist level or seeding the instability. Because of the discrete nature of fluxons, the system is not always 


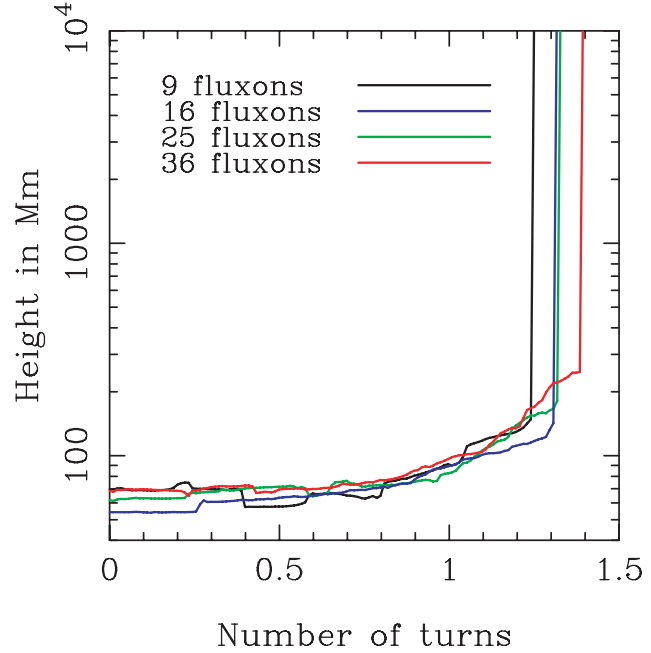

Figure 4. Flux rope remains well confined as it is twisted until it herniates rapidly through the arcade. The data shown are for systems with one unit of flux in the arcade.

symmetric, and this probably accounts for some uncertainty in the critical twist. There is not always a consistent trend with fluxon density, and so there may be other reasons behind this behavior.

The energy of the final erupted state is less than the energy of the confined flux rope (Figure 5). Note that the presence of the open boundary does not skew these results. The magnetic energy that escapes through the boundary would not be available to drive the CME in any case because it is present beyond the transition to the solar wind. In the latter stages of the expansion, the twist per unit length in the flux rope is small, and hence the free energy is low. It is the initial expansion that drives the CME, not the later expansion. Compared to the physical case, we overestimate the final magnetic energy of the system because our boundary is much farther out than the transition. A significant amount of energy is available to drive a CME, even without reconnection.

This simulation allows us to put a strong upper and lower bound on the amount of energy that is released with the eruption. Of the free energy injected, $12.5 \%$ is lost after herniation. The energy calculated in the ring field after herniation is an overestimate, and the energy in the flux rope and the arcade is an underestimate for the following reasons. The ring field was added so that all of the last fluxels were in that ring. As stated earlier, we do not trust the energy calculation for the ring field, especially considering that before herniation less than $1 \%$ of the system's energy was in the ring field compared to $\sim 40 \%$ after herniation. Also, on a spherical solar surface, the ring field would be farther away than the disk limb if the flux rope were at disk center, and consequently would not be highly sheared away from radial after the eruption, so it would not store much more energy than it had initially.

Because of the unreliability of the final energy in the ring field, we also looked at the energy in only the flux rope and the arcade. In this partial system, the final energy is less than the initial potential energy in part because some of the energy is carried through the open boundary and lost from the calculation, and in part because this limited system does not account for any background solar field that may be deformed by a CME.

Despite these effects, we are able to conclude that a significant amount of energy is released and could be used to drive an impulsive CME. The best way to resolve the energy would be

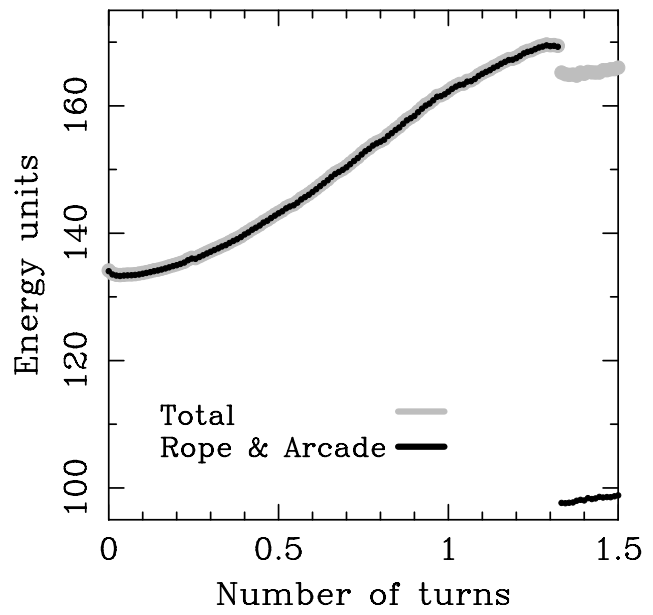

Figure 5. Typical energy plot for the simulations run. This one shows the energy evolution of a 25 fluxon flux rope with a one-unit flux arcade. As the flux rope is twisted under the arcade, the energy increases steadily. Once the system herniates, the energy loss is substantial. Note that the energy in the flux rope and arcade only (black line) drops to lower than the initial level. This is because most of the energy in the system at that point is in the outer ring fluxons which are highly stressed toward the horizontal.

to run a similar system as a full-Sun simulation in spherical geometry so that there are no last fluxons, and include an estimate of the energy outside the upper boundary with a force-free field extrapolation. This future work may be able to determine quantitatively how much magnetic energy is available - a figure which is highly dependent on the geometry of each event.

\section{DISCUSSION}

Our simulations show that reconnection is not necessary to initiate a CME and that impulsive CMEs may be possible without explosive reconnection. This theory is not new; it was originally published by Sturrock et al. (2001), who described the existence of metastable states, specifically a system similar to the one that we have studied. Since then, other solar physicists have studied this system computationally (Fan \& Gibson 2004; Török et al. 2004; Aulanier et al. 2005, etc.). The results from these studies show that a highly twisted flux rope can herniate through a confining magnetic arcade and reconnect into a plasmoid, causing an eruption. However, this is the first study of this system in the complete lack of reconnection. In general, our results agree with those of other research groups.

The fact that many of these simulations, including ours, agree that about 1.5 total turns are needed to herniate through an arcade implies that reconnection is not greatly important to the overall stability of the system. If it were, we would expect our ideal simulation to support significantly more twist, and therefore release more energy, than the dissipative simulations. The exact value of the critical twist depends on the configuration of the system: strength of the arcade, width of the flux rope, twist profile within the flux rope, etc. But even with these variables, we conclude that highly twisted flux ropes cannot easily be confined by an external field, even when the reconnection rate is extremely small.

Current research on the twist available photospheric fields indicates that there may be an excess of one full turn available in many active regions (Leka et al. 2005). This implies that many pre-eruptive active regions may be on the brink of an ideal instability when they flare or erupt, regardless of the eventual trigger mechanism. 
Our results together with the results of Wolfson \& Low (1992), Wolfson (1993), and Choe \& Cheng (2002) can finally put to rest the concerns that the Aly-Sturrock conjecture have created over the initiation of CMEs. Wolfson \& Low (1992) showed that a closed force-free field can have more energy than a partially open field with the same lower boundary field distribution and Choe \& Cheng (2002) proved that there are force-free fields that have more energy in the closed state than the open one. In the present work, we have not only confirmed that it is possible for a closed force-free system to store more energy than a partially opened erupted state; we have also, for the first time, demonstrated a mechanism by which an eruption may liberate that energy without the need for magnetic reconnection or other nonmagnetic confinement of the initial plasma. At the beginning of the century there was "still no model which demonstrates that a partly open magnetic field can be achieved solely by a loss of ideal MHD equilibrium or stability" (Forbes 2000). Happily, this statement is no longer true.

This work was funded by NASA's LWS-TR\&T program. FLUX is open source software available from http://flux.boulder.swri.edu. Thanks to the PDL development team http://pdl.perl.org. We also owe thanks to Spiro Antiochos, Bernhard Kliem, and Zoran Mikic for valuable discussions of the fluxon technique, and to Hugh Hudson for constructive comments on the paper.

\section{REFERENCES}

Aly, J. J. 1984, ApJ, 283, 349

Aly, J. J. 1991, ApJ, 375, L61

Antiochos, S. K., DeVore, C. R., \& Klimchuk, J. A. 1999, ApJ, 10, 485

Aulanier, G., Démoulin, P., \& Grappin, R. 2005, A\&A, 430, 1067

Berger, T. E., et al. 2008, ApJ, 676, L89
Choe, G. S., \& Cheng, C. Z. 2002, ApJ, 574, L179

DeForest, C. E., \& Kankelborg, C. C. 2007, Journal of Atmospheric and Terrestrial Physics, 69, 116

Fan, Y., \& Gibson, S. E. 2004, ApJ, 609, 1123

Fong, B., Low, B. C., \& Fan, Y. 2002, ApJ, 571, 987

Forbes, T. G. 2000, J. Geophys. Res., 105, 23153

Gibson, S. E., \& Low, B. C. 1998, ApJ, 493, 460

Gold, T., \& Hoyle, F. 1960, MNRAS, 120, 89

Hundhausen, A. J., Stanger, A. L., \& Serbicki, S. A. 1994, in Solar Dynamic Phenomena and Solar Wind Consequences, Vol. 373, ed. J. J. Hunt (Noordwijk: ESA), 409

Klimchuk, J. A. 2001, AGU Geophysical Monograph, 125, 2001

Kohl, J. L., et al. 1997, Sol. Phys., 175, 613

Leka, K. D., Fan, Y., \& Barnes, G. 2005, ApJ, 626, 1091

Low, B. C. 1986, ApJ, 307, 205

Low, B. C. 1990, ARA\&A, 28, 491

Low, B. C. 1994, Phys. Plasmas, 1, 1684

Low, B. C. 1996, Sol. Phys., 167, 217

Low, B. C. 2001, J. Geophys. Res., 106, 25141

Moore, R. L., \& Roumeliotis, G. 1992, in IAU Colloq. 133, Triggering of Eruptive Flares-Destabilization of the Preflare Magnetic Field Configuration, Eruptive Solar Flares, ed. Z. Svestka, B. V. Jackson, \& M. E. Machado (Berlin: Springer), 69

Parker, E. N. 1960, ApJ, 132, 175

Parker, E. N. 1963, ApJS, 8, 177

Petschek, H. E. 1964, in The Physics of Solar Flares, Magnetic Field Annihilation, ed. W. N. Hess (Washington, DC: NASA), 425

Priest, E. R. 1982, Solar Magnetohydrodynamics (Dordrecht: D. Reidel)

Priest, E. R., Hood, A. W., \& Anzer, U. 1989, ApJ, 344, 1010

Ridgway, C., Priest, E. R., \& Amari, T. 1991, ApJ, 367, 321

Roussev, I. I., Forbes, T. G., Gombosi, T. I., Sokolov, I. V., DeZeeuw, D. L., \& Birn, J. 2003, ApJ, 588, L45

Sturrock, P. A. 1991, ApJ, 380, 655

Sturrock, P. A., Weber, M., Wheatland, M. S., \& Wolfson, R. 2001, ApJ, 548, 492

Titov, V. S., \& Démoulin, P. 1999, A\&A, 351, 707

Török, T., Kliem, B., \& Titov, V. S. 2004, A\&A, 413, L27

van Ballegooijen, A. A., \& Martens, P. C. H. 1989, ApJ, 343, 971

Wolfson, R. 1993, ApJ, 419, 382

Wolfson, R., \& Low, B. C. 1992, ApJ, 391, 353

Zhang, M., \& Low, B. C. 2004, ApJ, 600, 1043 